\title{
Epidermolytic Ichthyosis Sine Epidermolysis
}

\author{
Marina Eskin-Schwartz, MD, PhD, *† Marianna Drozhdina, PhD, $\neq$ Ofer Sarig, PhD, * Andrea Gat, MD, $\S$

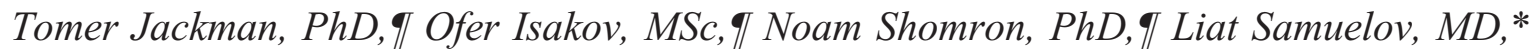 \\ Natalia Malchin, PhD, * Alon Peled, BMedSc, *† Dan Vodo, BMedSc, *† Alain Hovnanian, MD, PhD, $\|^{* *}$ \\ Thomas Ruzicka, MD, †† Sergei Koshkin, MD, PhD, $\neq$ Robert M. Harmon, PhD, $\neq \neq$

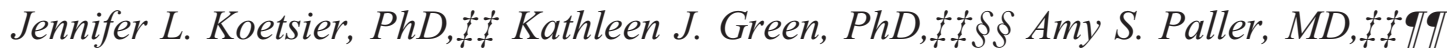 \\ and Eli Sprecher, $M D, P h D^{* \dagger}$
}

\begin{abstract}
Epidermolytic ichthyosis (EI) is a rare disorder of cornification caused by mutations in KRT1 and KRT10, encoding two suprabasal epidermal keratins. Because of the variable clinical features and severity of the disease, histopathology is often required to correctly direct the molecular analysis. EI is characterized by hyperkeratosis and vacuolar degeneration of the upper epidermis, also known as epidermolytic hyperkeratosis, hence the name of the disease. In the current report, the authors describe members of 2 families presenting with clinical features consistent with EI. The patients were shown to carry classical mutations in $K R T 1$ or KRT10, but did not display epidermolytic changes on histology. These observations underscore the need to remain aware of the limitations of pathological features when considering a diagnosis of EI.
\end{abstract}

Key Words: epidermolytic ichthyosis, KRT1, KRT10

(Am J Dermatopathol 2017;39:440-444)

\section{INTRODUCTION}

Epidermolytic ichthyosis (EI; MIM 113800), formerly known as epidermolytic hyperkeratosis or bullous congenital ichthyosiform erythroderma, is a rare and clinically heterogeneous disorder of cornification. ${ }^{1}$ EI is usually inherited in a dominant fashion, but rarely is recessive (MIM 113800) or semidominant, and is caused by mutations in KRT1 and $K R T 10$, which encode the 2 major suprabasal keratins, keratin

From the *Department of Dermatology, Tel Aviv Sourasky Medical Center, Tel Aviv, Israel; $\uparrow$ Department of Human Molecular Genetics \& Biochemistry, Sackler Faculty of Medicine, Tel Aviv University, Tel Aviv, Israel; tDepartment of Dermatology, Kirov State Medical Academy, Kirov, Russia; $\S$ Department of Pathology, Tel Aviv Sourasky Medical Center, Tel Aviv, Israel; $\uparrow$ Department of Cell and Developmental Biology, Sackler Faculty of Medicine, Tel Aviv University, Tel Aviv, Israel; |IINSERM UMR 1163, Imagine Institute, University Paris Descartes Sorbonne Cité, Paris, France; **Department of Genetics, Necker hospital for sick children, APHP, Paris, France; $\dagger$ † Department of Dermatology and Allergology, Ludwig-MaximilianUniversity, Munich, Germany; and Departments of + Pathology, $\S \S$ Dermatology, and $\uparrow \uparrow$ Department of Pediatrics, Northwestern University Feinberg School of Medicine, Chicago, IL.

Supported in part by a donation of the Ram family.

The authors declare no conflicts of interest.

Reprints: Eli Sprecher, MD, PhD, Department of Dermatology, Tel Aviv Medical Center, 6 Weizmann Street, Tel Aviv 64239, Israel (e-mail: elisp@tlvmc.gov.il).

Copyright (C) 2017 Wolters Kluwer Health, Inc. All rights reserved.
1 and keratin 10 , respectively. ${ }^{2-4} \mathrm{EI}$ is characterized by blistering in early life followed by generalized ichthyosis of varying severity, sometimes associated with diffuse palmoplantar keratoderma. ${ }^{1}$ Rarer variants of EI include annular (MIM 607602) and nevoid (MIM 162900) forms of the disease. In addition, mutations in KRT1 and KRT10 have been associated with a variety of non-EI phenotypes such as ichthyosis with confetti (MIM 609165) ${ }^{5}$ and ichthyosis hystrix of CurthMacklin (MIM 146590). ${ }^{6}$

Epidermolytic hyperkeratosis, the histopathological hallmark of EI, is characterized by vacuolar degeneration of suprabasal keratinocytes and coarse keratohyalin granules in the thickened granular layer, often associated with intracytoplasmic and perinuclear eosinophilic inclusions, which reflect tonofilaments clumping on electron microscopy. ${ }^{7}$

Because of the variable clinical manifestations of mutations in KRT1 and $K R T 10^{1}$ and the fact that EI shares many clinical features with other disorders of cornification, ${ }^{8}$ histopathology is often used to direct molecular analyses in cases of suspected EI. ${ }^{7}$ Here, we present 2 cases clinically consistent with EI and caused by classical mutations in KRTI and KRT10, but without epidermolytic changes on initial histological analysis.

\section{MATERIALS AND METHODS}

\section{Patients}

The patients and their healthy family members provided written and informed consent to participate in this study according to a protocol approved by the institutional review board at each site and by the Israel National Committee for Human Genetic Studies in adherence with the Helsinki principles. Skin biopsies were obtained from clinically affected areas for histological examination and immunostaining. DNA was extracted from peripheral blood leukocytes using the 5 Prime ArchivePure DNA Blood Kit (5 Prime Inc, Gaithersburg, MD).

\section{Immunohistochemical Studies}

Four $\mu \mathrm{m}$ paraffin-embedded sections were kept overnight at $60^{\circ} \mathrm{C}$ and deparaffinized. After permeabilization and antigen retrieval sections were blocked in $1 \%$ bovine serum albumin, $2 \%$ normal goat serum and incubated with primary antibodies, as previously described. ${ }^{9}$ Primary antibodies used 
were mouse monoclonal 4B2 (anti-desmoglein $1^{10} ; 1: 100$ dilution), mouse monoclonal 11-5F (anti-desmoplakin, gift from Dr. David Garrod, 1:100 dilution), and chicken polyclonal 1407 (anti-plakoglobin, ${ }^{11}$ diluted 1:1000). Secondary antibody staining was performed for 30 minutes at $37^{\circ} \mathrm{C}$ using Alexa Fluor anti-mouse, anti-rabbit, or anti-chicken secondary antibodies (1:300 dilution; Life Technologies/Invitrogen, Carlsbad, CA).

\section{Exome Sequencing}

Exome sequencing of individuals I-1, II-1, III-2, III-3, and IV-1 from family 1 (case 1) was performed by Otogenetics corporation using in-solution hybridization with Agilent AV5 + UTR Exome (71 Mb) version 4.0 (Agilent, Santa Clara) followed by massively parallel sequencing (Illumina HiSeq2000) with 100-bp paired-end reads. In the case of family 2, exome sequencing was performed by BGI Tech Solutions Ltd using in-solution hybridization with SureSelect All Exon $51 \mathrm{Mb}$ Version 4.0 followed by massively parallel sequencing (Illumina HiSeq2000) with 100-bp paired-end reads. Reads were aligned to the Genome Reference Consortium Human Build 37 (GRCh37/hg19) using BurrowsWheeler Aligner (BWA). ${ }^{12}$ Exome sequencing data analysis was performed as previously described. ${ }^{9}$

\section{Sanger Sequencing}

Genomic DNA was polymerase chain reaction amplified using the oligonucleotide primer pair: $5^{\prime}$-TCAACTGAACAAGG-3' and 5'-TACTCTACCCTCTCT-3', spanning KRT10 exon 6. Coding sequences and flanking intronic boundaries of KRT1 were polymerase chain reaction amplified as previously described. ${ }^{13}$ Gel-purified (QIAquick gel extraction kit; QIAGEN, Hilden, Germany) amplicons were subjected to bidirectional DNA sequencing with the BigDye terminator system on an ABI Prism 3100 sequencer (Applied Biosystems, NY).

\section{RESULTS}

\section{Family 1}

A four-generation kindred of Russian descent (Fig. 1A) presented with localized symmetric well-defined hyperkeratotic plaques which first developed at 5-6 years of age. The lesions were most prominent on the elbows, knees, hips, and dorsal feet, but in some cases also involved flexural areas such as axillae and groin. Some of the lesions clinically resembled plaque-type psoriasis. There was no history of blistering or skin fragility (Fig. 1B).

Histologically, psoriasiform hyperplasia with mild papillomatosis, minimal spongiosis, hypogranulosis, parakeratosis, and superficial perivascular lymphocytic infiltrate were observed. Small foci of intercellular separation in the spinous layer without gross epidermolytic changes or overt acantholysis were present (Fig. 1C).

Deep sequencing followed by validation through direct sequencing revealed that all affected members of family 1 harbor a heterozygous $\mathrm{G}>\mathrm{C}$ transversion at position 1322 of the KRT10 gene cDNA (Fig. 1D). The mutation is predicted to result in the substitution of a proline residue for a conserved arginine amino acid at position 441 of the amino acid sequence of the KRT10 protein (p.R441P). The mutation, which has been previously reported in a case of EI, ${ }^{14}$ was
FIGURE 1. Clinical and molecular features of family 1. A, Family pedigree. Black symbols denote affected individuals; (B) clinical features include well-demarcated hyperkeratotic plaques in the axillae, knees, ankles, and feet; (C) histological features comprise psoriasiform hyperplasia with mild papillomatosis, minimal spongiosis, hypogranulosis, parakeratosis, and superficial perivascular lymphocytic infiltrate. Small foci of intercellular separation in the spinous layer are present (hematoxylin and eosin, X200); (D) direct sequencing revealed a heterozygous $C .1322 \mathrm{G}>\mathrm{C}$ (p.R441P) mutation in KRT10 in the affected family members (lower panel). The wild-type (WT) sequence is given for comparison. The position of the mutation is underlined.

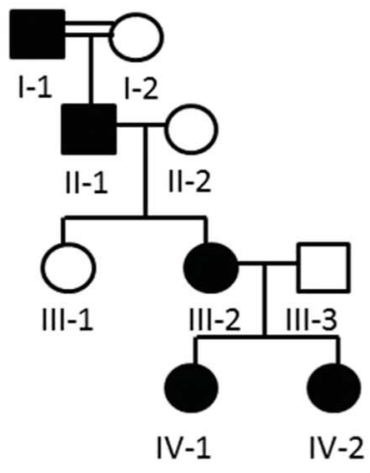

A

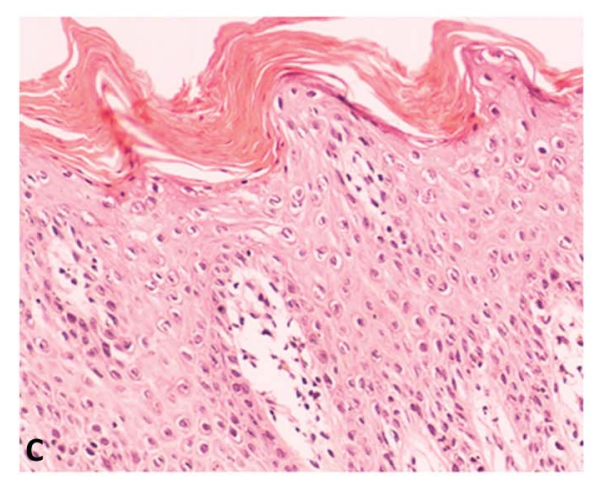

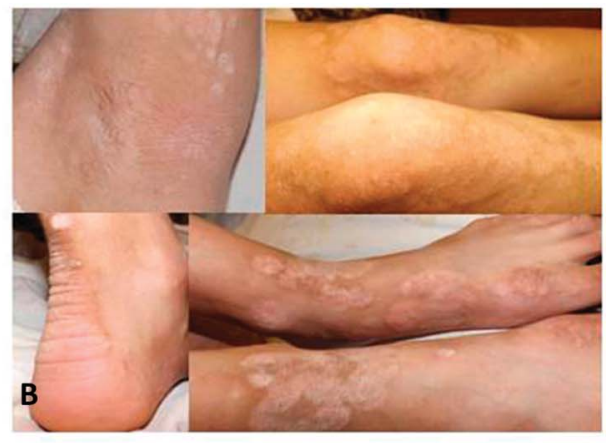

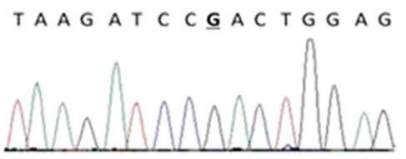

WT/WT

TA A A T C C $A C T G G A G$

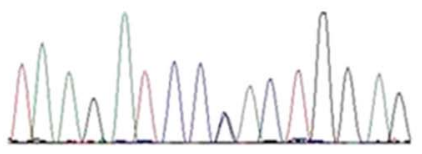

c. $1322 \mathrm{G}>\mathrm{C} / \mathrm{WT}$ 
absent in the nonaffected members of the family and was absent from all available public databases (ESP, UCSC, NCBI, HGMD, Ensembl, 1000 genomes).

\section{Family 2}

A 2-year-old boy, the only child of unrelated healthy parents of mixed Northern European descent, was born with generalized hypotrichosis, erythroderma, and skin peeling (Fig. 2A). Histopathological analysis of a skin biopsy at 2 days of age revealed cell-cell dissociation throughout the entire epidermis with foci of acantholysis (Fig. 2B). During the first 9 months of life, he also had failure to thrive and recurrent Staphylococcus aureus infections of the skin and blood, necessitating recurrent hospitalizations. Immunostaining for LEKTI, performed because of suspected Netherton syndrome, was normal (data not shown). Given the acantholysis, erythroderma, and hypotrichosis suggestive of a desmosomal defect, ${ }^{15}$ we scrutinized all coding sequences and intronic boundaries of a number of genes encoding desmosomal proteins, including $D S G 1, D S P$, JUP, PKP1, and CDSN, previously associated with similar clinical and/or pathological features, ${ }^{15}$ as well as DSC1. No pathogenic mutations were identified.

Whole-exome sequencing then revealed a novel c. $562 \mathrm{~A}>\mathrm{C}$ missense mutation in $K R T 1$, predicted to result in the substitution of asparagine for histidine at position 188 of the protein sequence (p.N188H) (Fig. 2C). The mutation was absent in both parents, suggesting that it appeared de novo in the affected child or resulted from gonadal mosaicism. Supporting the pathogenicity of this mutation, it was found to affect a highly conserved residue (Conseq $=9$, range $=1-9 ;$ http://conseq.tau.ac.il/) and is predicted to be pathogenic by both SIFT $(0 \text {, range } 1-0)^{16}$ and POLYPHEN (1, range $0-1)^{17}$ software, used to estimate the possible impact of an amino acid substitution on the protein function. Finally, a number of other mutations have been reported to affect the same residue. ${ }^{18-22}$

A systematic inspection of the deep sequencing data failed to reveal another pathogenic mutation to explain the acantholytic changes seen on histology, with the possible exception of a heterozygous missense mutation found in the patient and his father in the $A 2 M L 1$ gene, encoding a major autoantigen in paraneoplastic pemphigus. ${ }^{23}$ The A2ML1 sequence change, c. $3145 \mathrm{C}>\mathrm{G}$, is predicted to lead to the substitution of a poorly conserved glutamine for glutamic acid (p.Q1049E), casting doubt as to its significance (not shown). In an attempt to provide an explanation for the loss of cellcell adhesion in the epidermis in the patient, we stained the patient biopsy for a number of adhesion molecules. We observed dramatically diminished expression of desmoglein 1, desmoplakin, and plakoglobin (Fig. 3). Throughout the first year of life, the patient had progressively fewer cutaneous $S$. aureus infections, but his skin grew increasingly more keratotic with extensive palmoplantar thickening. He continued to show blistering, prompting performance of a second biopsy at 2 years of age. Biopsy sections now revealed typical epidermolytic changes and hyperkeratosis (Fig. 2D).

\section{DISCUSSION}

The clinical spectrum of EI is broad, ranging from severe generalized disease, characterized by blistering in early
FIGURE 2. Clinical and molecular features in family 2. A, Clinical features included erythroderma associated with diffuse skin peeling; (B) a skin biopsy obtained at 2 days of age showed cell-cell disadhesion and foci of acantholysis (arrows) (hematoxylin and eosin, X40); (C) Sanger sequencing revealed a heterozygous c.562A $>$ C (p. $\mathrm{N188H})$ mutation in KRT1 in the affected child (upper panel) which was absent in his father (middle panel) and mother (lower panel). The position of the mutation is underlined; (D) a skin biopsy obtained at age 2 years showed epidermolytic changes in the upper epidermal layers (hematoxylin and eosin, $\times 40$ ).
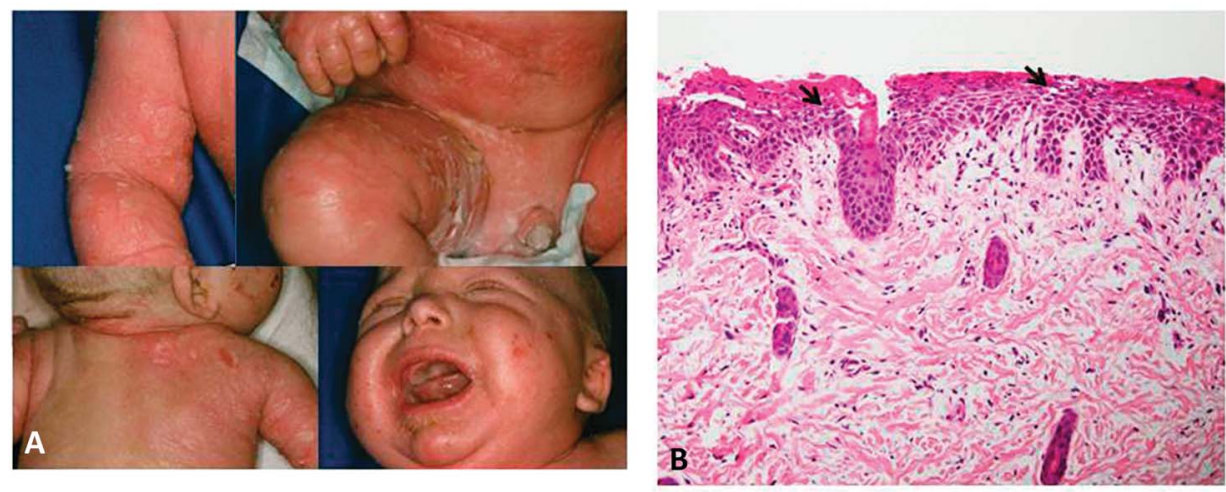

Patient

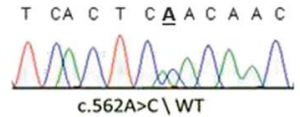

Father

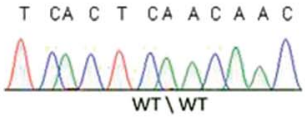

$T C A C T$ CA A C A A C

C

Mother

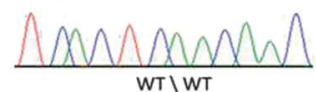

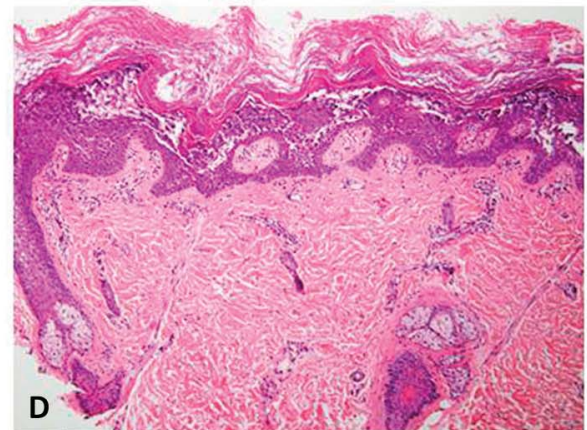



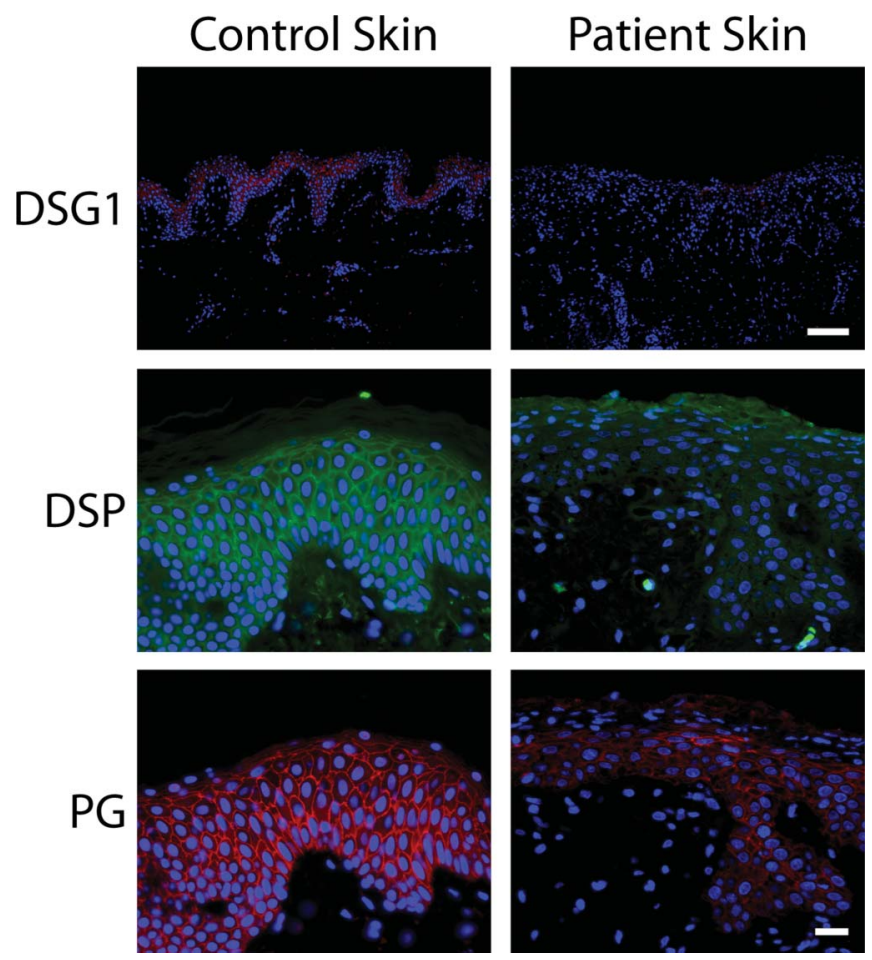

FIGURE 3. Immunostaining for desmosomal proteins. Skin sections from the biopsy obtained from the patient at 2 days of age and from a control subject were immunostained for desmosomal components desmoglein 1 (DSG1), desmoplakin (DSP), and plakoglobin (PG). Top panels, staining for desmoglein 1 (DSG1, red) captured at 10× magnification, calibration bar $100 \mu \mathrm{m}$. Nuclei were visualized with $4^{\prime} 6^{\prime}$-diamidino-2-phenylindole (DAPI). Middle and lower panels, staining for desmoplakin (DSP) and PG, respectively, captured at $40 \times$ magnification, calibration bar $20 \mu \mathrm{m}$. Desmoglein 1 and desmoplakin are drastically reduced in the patient epidermis, and PG staining is also weaker in the patient skin compared with the control biopsy.

life and widespread ichthyosis in adult life to mild phenotypes such as isolated palmoplantar keratoderma. ${ }^{1}$ The disease is caused by mutations affecting conserved regions primarily located at the beginning and end of the central alpha-helical rod of keratin molecules. ${ }^{4}$ Absence of epidermolytic changes is typical of disorders of cornification resulting from mutations affecting KRT1 or KRT10 domains located outside those regions. ${ }^{6,24}$ In contrast, here, we report absence of epidermolytic changes despite the presence of mutations affecting highly conserved regions of the rod domain.

The mutation detected in family 1 has been previously reported in a mild case of $\mathrm{EI}^{25}$ Interestingly, the phenotypes reported in our patient and in this previous report were remarkably similar both at the clinical and histopathological levels. ${ }^{25}$ Although it is highly conserved, it is possible that the residue affected by this recurrent mutation does not mediate keratin dimer formation but rather is involved in late differentiation-related events. ${ }^{25}$

In contrast with this first case, the patient of family 2 displayed a very severe phenotype featuring erythroderma, blistering, and peeling of the entire surface of the skin. Histology was remarkable for evidence of compromised cellcell adhesion, which correlated with decreased expression of several major desmosomal proteins, suggesting that the p.N188H mutation in KRT1 may alter keratinocyte cell-cell adhesion. Because other mutations have been shown to affect the p.N188 residue (p.N188S, p.N188T, and p.N188K) ${ }^{18-22}$ and to be associated with epidermolysis, it is tempting to suggest that the effect of the p.R188H mutation is specifically due to the nature of the substitution.

The pathomechanism underlying the effect of the p.N188H mutation on cell-cell adhesion remains to be investigated. Desmoplakin connects the intermediate filament network to the desmosomal plaque. ${ }^{26}$ In the absence of functional keratins, desmosomal adhesion is compromised. ${ }^{27}$ Desmoplakin has been shown to interact with the keratin 1 head domain. $^{28,29}$ As p.N188H affects a residue located within the $1 \mathrm{~A}$ domain of the keratin 1 protein, it is unclear whether the mutation leads to desmosomal destabilization through a direct effect on desmoplakin-keratin 1 interaction, as shown for other keratins, ${ }^{30}$ or exert a deleterious effect on desmosomal stability indirectly by interfering with keratin dimerization, which has also been shown to be critical for desmoplakin interaction with intermediate filaments in some experimental systems. ${ }^{30,31} \mathrm{Of}$ note, although no epidermolytic changes were visible in the biopsy obtained perinatally in the family 2 patient, a biopsy obtained in early infancy revealed vacuolar degeneration of the upper epidermal layers. The coexistence in the same individual of epidermolytic changes and acantholysis has been reported in the past in EI but is likely to be rare. ${ }^{32}$ Of note, cell-cell separation (but not overt acantholysis) was noticed in case 1 .

In summary, the present report and a previous study ${ }^{14}$ call for caution when interpreting the lack of epidermolytic changes in a case clinically suggestive of EI.

\section{REFERENCES}

1. DiGiovanna JJ, Bale SJ. Clinical heterogeneity in epidermolytic hyperkeratosis. Arch Dermatol. 1994;130:1026-1035.

2. Nousbeck J, Padalon-Brauch G, Fuchs-Telem D, et al. Semidominant inheritance in epidermolytic ichthyosis. $J$ Invest Dermatol. 2013;133: 2626-2628.

3. Muller FB, Huber M, Kinaciyan T, et al. A human keratin 10 knockout causes recessive epidermolytic hyperkeratosis. Hum Mol Genet. 2006;15: $1133-1141$.

4. Lane EB, McLean WH. Keratins and skin disorders. J Pathol. 2004;204: 355-366.

5. Choate KA, Lu Y, Zhou J, et al. Mitotic recombination in patients with ichthyosis causes reversion of dominant mutations in KRT10. Science. 2010;330:94-97.

6. Sprecher E, Ishida-Yamamoto A, Becker OM, et al. Evidence for novel functions of the keratin tail emerging from a mutation causing ichthyosis hystrix. J Invest Dermatol. 2001;116:511-519.

7. Bergman R, Khamaysi Z, Sprecher E. A unique pattern of dyskeratosis characterizes epidermolytic hyperkeratosis and epidermolytic palmoplantar keratoderma. Am J Dermatopathol. 2008;30:101-105.

8. Oji V, Tadini G, Akiyama M, et al. Revised nomenclature and classification of inherited ichthyoses: results of the first ichthyosis Consensus Conference in Soreze 2009. J Am Acad Dermatol. 2010;63:607-641.

9. Samuelov L, Sarig O, Harmon RM, et al. Desmoglein 1 deficiency results in severe dermatitis, multiple allergies and metabolic wasting. Nat Genet. 2013;45:1244-1248.

10. Dusek RL, Getsios S, Chen F, et al. The differentiation-dependent desmosomal cadherin desmoglein 1 is a novel caspase- 3 target that regulates apoptosis in keratinocytes. J Biol Chem. 2006;281:3614-3624. 
11. Gaudry CA, Palka HL, Dusek RL, et al. Tyrosine-phosphorylated plakoglobin is associated with desmogleins but not desmoplakin after epidermal growth factor receptor activation. J Biol Chem. 2001;276:24871-24880.

12. Li H, Durbin R. Fast and accurate long-read alignment with BurrowsWheeler transform. Bioinformatics. 2010;26:589-595.

13. Whittock NV, Eady RA, McGrath JA. Genomic organization and amplification of the human epidermal type II keratin genes K1 and K5. Biochem Biophys Res Commun. 2000;274:149-152.

14. Mirza H, Kumar A, Craiglow BG, et al. Mutations affecting keratin 10 surface-Exposed residues Highlight the Structural Basis of phenotypic Variation in epidermolytic ichthyosis. J Invest Dermatol. 2015;135:3041-3050.

15. Samuelov L, Sprecher E. Inherited desmosomal disorders. Cell Tissue Res. 2015;360:457-475.

16. Kumar P, Henikoff S, Ng PC. Predicting the effects of coding nonsynonymous variants on protein function using the SIFT algorithm. Nat Protoc. 2009;4:1073-1081.

17. Adzhubei IA, Schmidt S, Peshkin L, et al. A method and server for predicting damaging missense mutations. Nat Methods. 2010;7:248-249.

18. McLean WH, Eady RA, Dopping-Hepenstal PJ, et al. Mutations in the rod $1 \mathrm{~A}$ domain of keratins 1 and 10 in bullous congenital ichthyosiform erythroderma (BCIE). $J$ Invest Dermatol. 1994;102:24-30.

19. Arin MJ, Longley MA, Kuster W, et al. An asparagine to threonine substitution in the 1A domain of keratin 1: a novel mutation that causes epidermolytic hyperkeratosis. Exp Dermatol. 1999;8:124-127.

20. Nomura K, Umeki K, Hatayama I, et al. Phenotypic heterogeneity in bullous congenital ichthyosiform erythroderma: possible somatic mosaicism for keratin gene mutation in the mildly affected mother of the proband. Arch Dermatol. 2001;137:1192-1195.

21. Arin MJ, Oji V, Emmert S, et al. Expanding the keratin mutation database: novel and recurrent mutations and genotype-phenotype correlations in 28 patients with epidermolytic ichthyosis. Br J Dermatol. 2011;164:442-447.

22. Lee DY, Ahn KS, Lee CH, et al. Two novel mutations in the keratin 1 gene in epidermolytic hyperkeratosis. J Invest Dermatol. 2002;119:976-977.
23. Schepens I, Jaunin F, Begre N, et al. The protease inhibitor alpha-2macroglobulin-like-1 is the p170 antigen recognized by paraneoplastic pemphigus autoantibodies in human. PLoS One. 2010;5:e12250.

24. Kimonis V, DiGiovanna JJ, Yang JM, et al. A mutation in the V1 end domain of keratin 1 in non-epidermolytic palmar-plantar keratoderma. J Invest Dermatol. 1994;103:764-769.

25. Mirza H, Kumar A, Craiglow BG, et al. Mutations affecting keratin 10 surface-exposed residues highlight the structural basis of phenotypic variation in epidermolytic ichthyosis. $J$ Invest Dermatol. 2015;135:3041-3050.

26. Simpson CL, Patel DM, Green KJ. Deconstructing the skin: cytoarchitectural determinants of epidermal morphogenesis. Nat Rev Mol Cell Biol. 2011;12:565-580.

27. Bar J, Kumar V, Roth W, et al. Skin fragility and impaired desmosomal adhesion in mice lacking all keratins. $J$ Invest Dermatol. 2014;134: $1012-1022$.

28. Meng JJ, Bornslaeger EA, Green KJ, et al. Two-hybrid analysis reveals fundamental differences in direct interactions between desmoplakin and cell type-specific intermediate filaments. J Biol Chem. 1997;272:2149521503.

29. Kouklis PD, Hutton E, Fuchs E. Making a connection: direct binding between keratin intermediate filaments and desmosomal proteins. $J$ Cell Biol. 1994;127:1049-1060.

30. Fontao L, Favre B, Riou S, et al. Interaction of the bullous pemphigoid antigen 1 (BP230) and desmoplakin with intermediate filaments is mediated by distinct sequences within their $\mathrm{COOH}$ terminus. Mol Biol Cell. 2003; 14:1978-1992.

31. Kroger C, Loschke F, Schwarz N, et al. Keratins control intercellular adhesion involving PKC-alpha-mediated desmoplakin phosphorylation. J Cell Biol. 2013;201:681-692.

32. Ross R, DiGiovanna JJ, Capaldi L, et al. Histopathologic characterization of epidermolytic hyperkeratosis: a systematic review of histology from the national registry for ichthyosis and related skin disorders. $J$ Am Acad Dermatol. 2008;59:86-90. 